IJEMD-M, 1 (1) (2022) $48-65 \quad \underline{\text { https://doi.org/ } 10.54938 / \text { ijemdm.2022.01.1.18 }}$
International Journal of Emerging Multidiciplinaries
Mathematics
$\begin{gathered}\text { Research Paper } \\ \text { Journal Homepage: } \text { www.ijemd.com } \\ \text { ISSN (print): } 2790-1998\end{gathered}$

\title{
Modeling and analysis of fractional TB model with Atangana- Baleanu derivative
}

\author{
Aatif Ali ${ }^{1, *}$, Quaid Iqbal ${ }^{1}$, Huma Gul ${ }^{1}$, Muhammad Nafees ${ }^{1}$ and Saeed Islam $^{1}$ \\ ${ }^{I}$ Department of Mathematics, Abdul Wali Khan University Mardan, 23200, KP, Pakistan. \\ *Corresponding author
}

\begin{abstract}
In recent years Atangana and Baleanu proposed a new fractional derivative with non-singular and non-local kernel, this paper formulate a fragmentary request numerical TB model with Atangana-Baleanu derivative. We inquired the basic reproduction number and assessment of boundary dependent on genuine information of Khyber Pakhtunkhwa Pakistan, Initially we present the fundamental properties of the model, the existence and uniqueness of the model is proved through fixed point theory. At last, the model is tackled mathematically through Adams-Bashforth Moulton technique. The mathematical results for the extended model of the elements of Tuberculosis is shown graphically to feature the actual conduct of the issue and the underlying conditions are presented. The graphical results clarify the impact of various boundaries. From the examination it is tracked down that fragmentary request gives more understanding with regards to the infection elements.
\end{abstract}

Keywords: Atangana-Baleanu fractional derivative; Tuberculosis (TB); Estimation; Simulation.

\section{Introduction}

Tuberculosis is one of the top ten causes of death worldwide, it is a bacterial infectious disease resulting from bacillus mycobacterium tuberculosis (MTB). It is basically divided into two categories pulmonary TB, which affects the lungs and extrapulmonary TB that invades other organs like brain, spine, kidneys, central nervous system or lymphatic system, TB can be transmitted from a patient through cough, spit, sneeze or speak, the main symptoms of TB includes chronic cough, blood-containing sputum, night sweats, fever and weight loss. It all started roughly 3 million years ago in East Africa. According to current WHO figures, roughly a quarter of the population of world is at risk of contracting tuberculosis (TB), with more than 10.4 million people infected and 1.7 million died due to TB in 2016 [1]. A tuberculosis infected person has a 5-15 percent lifetime 
danger of contracting the sickness. In Pakistan, the number of individuals diagnosed with tuberculosis (TB) is rising, with an estimated 27,000 new cases recorded each year, accounting for 87 percent of all new TB cases. According to current incidence data, Pakistan has a total TB case incidence rate of 525,000 cases per year, with 368,589 cases under treatment and around 56,000 fatalities related to TB per year. Bangladesh and South Africa are among the 30 nations with the highest TB burden in 2019 [2, 3]. Tuberculosis comes in three forms: active, military, and latent TB infection. Mathematical models are essential for understand the dynamics of diseases. In 1962, the first TB model was proposed by Waller et al. [4], which divided the entire population into three subgroups.

In 1967, a proportion dependent mathematical model of tuberculosis infection is developed [5]. Castillo et al. presented two-strain, age-structured, and time-delay TB infection models in [6, 7]. In [8], the authors studied Mathematical Analysis of the TB Model with Treatment via Caputo-Type Fractional Derivative. In [9] a new Hepatitis B model in light of asymptomatic carriers and vaccination study through Atangana-Baleanu derivative is analyzed. Liu et al [10] investigated TB dynamics with seasonality by using data to estimate model parameters. Robert investigates the impact of relapse and reinfection in the TB infective population in [11]. Kim et al. recently used real-world data from the Philippines population to develop a mathematical model with optimal TB management tactics [12]. Due to its memory and representation of hereditary qualities, fractional-order mathematical models are more prominent and valuable in evaluating real-world phenomena than integer-order models [13-17].The integer-order derivative does not investigate the dynamics among two points in real-world applications. Because of the limitations of integer order and local differentiation, [18] presents numerous concepts on differentials with non-local or fractional order and exponential Kernel. The newly presented Atangana-Baleanu derivative has been successfully used to a variety of real-world complex issues, as shown in [19-22]. This paper formulate a new AB fractional order model for tuberculosis infection. The concept of fixed point theory is used to show the existence and uniqueness of the TB model. The impact of various parameters will be explained using graphical data. The following section covers the fundamental definitions and results of the $\mathrm{AB}$ derivative. The numerical results for the dynamics of tuberculosis are shown graphically.

\section{Preliminaries}

We present some definitions of AB fractional derivative [23], which will be used in our proposed TB model,

Definition 1. Let $g \in H(a, b), b>a, \alpha \in[0,1]$, so in Caputo's (ABC) interpretation, the new fractional derivatives are,

$$
a^{A B C} D_{t}^{\alpha}(g(t))=\frac{B(\alpha)}{1-\alpha} \int_{a}^{t} g^{\prime}(y) E_{\alpha}\left[-\alpha \frac{(t-y)^{\alpha}}{1-\alpha}\right] d y
$$

Definition 2. The following is the definition of the fractional integral of the new fractional derivative with non-local kernel,

$$
a^{A B} I_{t}^{\alpha}(g(t))=\frac{1-\alpha}{\beta(\alpha)} g(t)+\frac{\alpha}{\beta(\alpha) \Gamma(\alpha)} \int_{a}^{t} g(y)(t-y)^{\alpha-1} d y
$$

Definition 3. If $g \in H^{1}(a, b), b>a, \alpha \in[0,1]$, and are not necessary differentiable, the new AB fractional derivative in Riemann-Liouville (ABR) sense, 


$$
a^{A B R} D_{t}^{\alpha}(g(t))=\frac{\beta(\alpha)}{1-\alpha} \frac{d}{d t} \int_{\alpha}^{t} g(y) E_{\alpha}\left[-\alpha \frac{(t-y)^{\alpha}}{1-\alpha}\right] d y
$$

Theorem 1. Let we have a function $g(t)$ which is continuous on [a, b] then the following holds,

$$
\left\|a^{A B R} D_{t}^{\alpha}(g(t))\right\|<\frac{B(\alpha)}{1-\alpha}\|g(y)\| \text { Where }\|g(x)\|=\max _{a \leq x \leq b}|g(y)| \text {. }
$$

Theorem 2. By ABC and ABR derivatives, the Lipchitz condition is satisfied as mentioned below:

$$
\left\|a^{A B C} D_{t}^{\alpha} g_{1}(t)-a^{A B C} D_{t}^{\alpha} g_{2}(t)\right\|<P\left\|g_{1}(t)-g_{2}(t)\right\|
$$

also for ABR derivative we have,

$$
\left\|a^{A B R} D_{t}^{\alpha} g_{1}(t)-a^{A B R} D_{t}^{\alpha} g_{2}(t)\right\|<P\left\|g_{1}(t)-g_{2}(t)\right\| \text {. }
$$

Theorem 3. The following FDEs,

$$
a^{A B C} D_{t}^{\alpha} g(t)=s(t)
$$

After the application of inverse Laplace transform and convolution result [23] , it gives a unique solution,

$$
g(t)=\frac{1-\alpha}{A B C(\alpha)} s(t)+\frac{\alpha}{A B C(\sigma) \Gamma(\alpha)} \int_{a}^{t} s(\xi)(t-\xi)^{\alpha-1} d \xi
$$

\section{TB model formulation with $\mathrm{AB}$ derivative}

To formulate the model, we divide the total human population $\mathrm{N}(\mathrm{t})$ in six groups. Susceptible is denoted by $\mathrm{S}(\mathrm{t})$, slowly and rapidly exposed classes are represented by $E_{1}(t)$ and $E_{2}(t)$ respectively, the recovered individuals are represented by $\mathrm{R}(\mathrm{t})$.TB infected by $\mathrm{I}(\mathrm{t})$ and treatment is denoted by $\mathrm{T}(\mathrm{t})$, a new fractional order $\mathrm{AB}$ derivative of order $\alpha$, satisfying $0<\alpha \leq 1$ is considered in this paper, through the following system,

$$
\begin{aligned}
{ }^{A B C} D_{t}^{\alpha} S & =\Lambda-\frac{\beta S I}{N}-\mu S \\
{ }^{A B C} D_{t}^{\alpha} E_{1} & =q \frac{\beta S I}{N}-\left(\mu+\varepsilon_{1}\right) E_{1}+(1-\eta) \delta T, \\
{ }^{A B C} D_{t}^{\alpha} E_{2} & =(1-q) \frac{\beta S I}{N}+\varepsilon_{1} E_{1}-\left(\mu+\varepsilon_{2}\right) E_{2}, \\
{ }^{A B C} D_{t}^{\alpha} I & =\varepsilon_{2} E_{2}+\eta \delta T-\left(\mu+\gamma+\alpha_{1}\right) I, \\
{ }^{A B C} D_{t}^{\alpha} T & =\gamma I-\left(\mu+\delta+\alpha_{2}+v\right) T, \\
{ }^{A B C} D_{t}^{\alpha} R & =v T-\mu R .
\end{aligned}
$$


With initial conditions,

$$
S(t)>0, \quad E_{1}(t) \geq 0, \quad E_{2}(t) \geq 0, \quad I(t) \geq 0, \quad R(t) \geq 0 .
$$

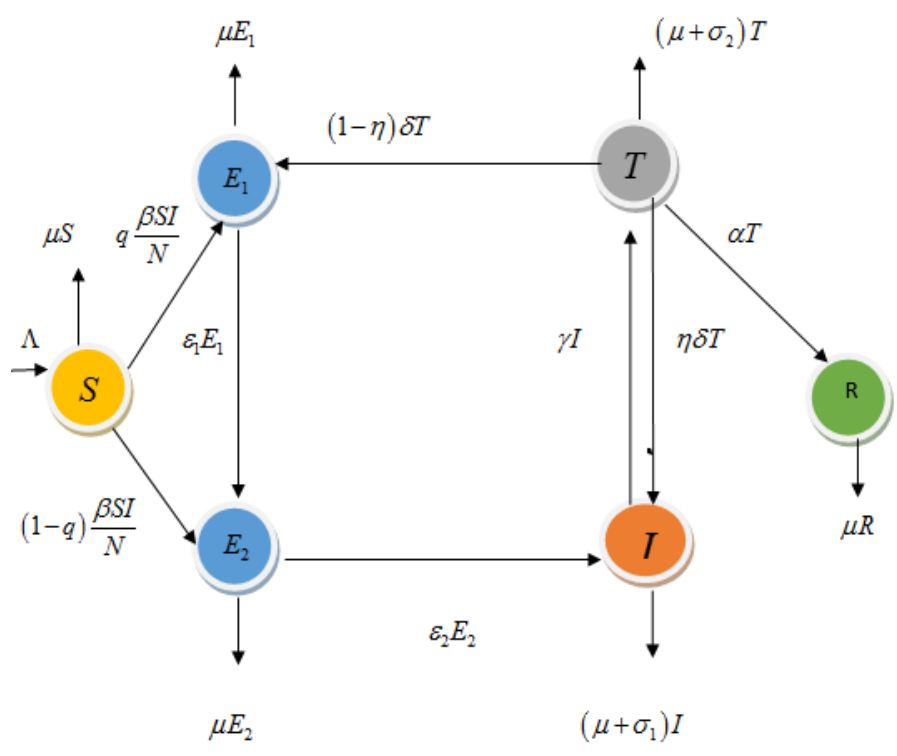

Schematic diagramme of TB model

In model (9), after powerful communication with infected individuals, a fraction

$q(0<q<1)$ of $S(t)$ move in to show exposed class $E_{1}$ and a fraction $(1-q)$ disclose straight to the exposed class $E_{2}$. In all classes, the parameter $(\Lambda)$ indicates the birth rate, the natural death rate is denoted by $\mu$ in all classes $\alpha_{1}$ and $\alpha_{2}$ are the disease death rates in $I$ and $T$ compartments. $\beta$ is the successful transmission coefficient, $\varepsilon_{1}$ is the alignment from compartment $E_{1}$ to $E_{2}$ and $\varepsilon_{2}$ represents the alignment from compartment $E_{2}$ to $\gamma$ and $I$ states the treatment rate of infected people. In the treatment compartment, the individual leave the class at a rate $\delta$ that leaves them with incomplete therapy, and depending on their level of healing, some of them $(\eta \delta T)$ re-enter the infected class $I$, while the others $((1-\eta) \delta T)$ return to the slow expose class $E_{1}$. The rate at which the people from treatment class enter the recovered class is represented by parameter $v$. The fraction of drug-resistant patients in the treated group is represented by the parameter $\eta(0<\eta<1)$ in the factor $(1-\eta) \delta$.In its basic sense, model (9) will be denoted as,

$$
p_{1}=\left(\mu+\varepsilon_{1}\right), p_{2}=\left(\mu+\varepsilon_{2}\right), p_{3}=\left(\mu+\gamma+\alpha_{1}\right), p_{4}=\left(\mu+\delta+\alpha_{2}+v\right)
$$

The DFE of the fractional TB model (9) is represented by $Z_{0}$ and is given as $Z_{0}=\left(\frac{\Lambda}{\mu}, 0,0,0,0,0\right)$ while the endemic equilibrium is denoted by $D_{1}=\left(S^{*}, E_{1}^{*}, E_{2}^{*}, I^{*}, T^{*}, R^{*}\right)$ and is given by, 


$$
\begin{aligned}
& S^{*}=\frac{N^{*}}{R_{0}}, \\
& E_{1}^{*}=\frac{1}{p_{1} p_{4}}\left(\frac{p_{4} q \beta}{R_{0}}+(1-\eta) \gamma \delta\right) I^{*}, \\
& E_{2}^{*}=\frac{1}{\varepsilon_{2} p_{4}}\left(p_{4} p_{3}-\eta \gamma \delta\right) I^{*}, \\
& T^{*}=\frac{\gamma}{p_{4}} I^{*}, \\
& R^{*}=\frac{v \gamma}{\mu p_{4}} I^{*}, \\
& I^{*}=\frac{p_{1} p_{4} \mu \varepsilon_{2}\left(R_{0}-1\right) N^{*}}{p_{4} \mu \varepsilon_{2} q \beta+\left(\mu\left(p_{1}\left(p_{3} p_{4}-\eta \gamma \delta\right)+\gamma \varepsilon_{2}\left(p_{1}+(1-\eta) \delta\right)\right)+p_{1} \varepsilon_{2}\left(v \gamma+p_{4} \mu\right)\right) R_{0}} .
\end{aligned}
$$

The basic reproduction number $R_{0}$, defined as "the number of secondary cases that one case would produce in a completely susceptible population" of the model is computed through the next-generation technique [24] presented as,

$$
R_{0}=\frac{\beta p_{4} \varepsilon_{2}\left(q \varepsilon_{1}+(1-q) p_{1}\right)}{p_{1} p_{2}\left(p_{3} p_{4}-\gamma \delta \eta\right)-\varepsilon_{1} \varepsilon_{2} \gamma \delta(1-\eta)}
$$

\section{Existence of Solution for TB model}

By the application of fixed point theory, we show the existence of the model solution in detail. It is difficult to determine the exact solution to model (9) due to the nonlinearity involved. However, if the existence of the model is proven, the model will have exact results under certain conditions. The system (9) can be structured as follows,

$$
\begin{aligned}
0^{A B C} D_{t}^{\alpha}[S(t)] & =p_{1}(t, S), \\
0^{A B C} D_{t}^{\alpha}\left[E_{1}(t)\right] & =p_{2}\left(t, E_{1}\right), \\
0^{A B C} D_{t}^{\alpha}\left[E_{2}(t)\right] & =p_{3}\left(t, E_{2}\right), \\
0^{A B C} D_{t}^{\alpha}[I(t)] & =p_{4}(t, I), \\
0^{A B C} D_{t}^{\alpha}[T(t)] & =p_{5}(t, T), \\
0^{A B C} D_{t}^{\alpha}[R(t)] & =p_{6}(t, R) .
\end{aligned}
$$

The following is a representation of the system (25) as a result of Theorem 3. 


$$
\begin{gathered}
S(t)-S(0)=\frac{(1-\alpha)}{A B(\alpha)} p_{1}(t, S)+\frac{\alpha}{A B(\alpha) \Gamma(\alpha)} \int_{0}^{t} p_{1}(\xi, S)(t-\xi)^{\alpha-1} d \xi \\
E_{1}(t)-E_{1}(0)=\frac{(1-\alpha)}{A B(\alpha)} p_{2}\left(t, E_{1}\right)+\frac{\alpha}{A B(\alpha) \Gamma(\alpha)} \int_{0}^{t} p_{2}\left(\xi, E_{1}\right)(t-\xi)^{\alpha-1} d \xi \\
E_{2}(t)-E_{2}(0)=\frac{(1-\alpha)}{A B(\alpha)} p_{3}\left(t, E_{2}\right)+\frac{\alpha}{A B(\alpha) \Gamma(\alpha)} \int_{0}^{t} p_{3}\left(\xi, E_{2}\right)(t-\xi)^{\alpha-1} d \xi, \\
I(t)-I(0)=\frac{(1-\alpha)}{A B(\alpha)} p_{4}(t, I)+\frac{\alpha}{A B(\alpha) \Gamma(\alpha)} \int_{0}^{t} p_{4}(\xi, I)(t-\xi)^{\alpha-1} d \xi \\
T(t)-T(0)=\frac{(1-\alpha)}{A B(\alpha)} p_{5}(t, T)+\frac{\alpha}{A B(\sigma) \Gamma(\alpha)} \int_{0}^{t} p_{5}(\xi, T)(t-\xi)^{\alpha-1} d \xi \\
R(t)-R(0)=\frac{(1-\alpha)}{A B(\alpha)} p_{6}(t, R)+\frac{\alpha}{A B(\alpha) \Gamma(\alpha)} \int_{0}^{t} p_{6}(\xi, R)(t-\xi)^{\alpha-1} d \xi .
\end{gathered}
$$

In the following theorem, we prove the Lipchitz condition.

Theorem 4. The $p_{1}$ fulfills the Lipchitz condition and contraction if the below inequality holds,

$$
0 \leq\left(\beta \omega_{1}+\mu\right)<1
$$

Proof. We would consider the functions $S$ and $S_{1}$ to show the result,

$$
\begin{aligned}
\left\|p_{1}(t, S)-p_{1}\left(t, S_{1}\right)\right\| & =\left\|-\frac{\beta I}{N}\left(S(t)-S\left(t_{1}\right)\right)-\mu\left(S(t)-S\left(t_{1}\right)\right)\right\| \\
& \leq \beta\|I(t)\|\left\|S(t)-S\left(t_{1}\right)\right\|+\mu\left\|S(t)-S\left(t_{1}\right)\right\| \\
& \leq\left\{\beta \omega_{1}+\mu\right\}\left\|S(t)-S\left(t_{1}\right)\right\| \\
& \leq \lambda_{1}\left\|S(t)-S\left(t_{1}\right)\right\|,
\end{aligned}
$$

Where $\lambda_{1}=\left\{\beta \omega_{1}+\mu\right\}$ and $\|I(t)\| \leq \omega_{1}$, which implies,

$$
\left\|p_{1}(t, S)-p_{1}\left(t, S_{1}\right)\right\| \leq \lambda_{1}\left\|S(t)-S\left(t_{1}\right)\right\| .
$$

As a result, the Lipchitz condition is satisfied. Additionally, if $0 \leq\left(\beta \omega_{1}+\mu\right)<1$ then a contraction implies. The rest of the cases, which are shown below, are analyzed in the same way: 


$$
\begin{aligned}
& \left\|p_{2}(t, E)-p_{2}\left(t, E_{1}\right)\right\| \leq \lambda_{2}\left\|E(t)-E\left(t_{1}\right)\right\|, \\
& \left\|p_{3}(t, E)-p_{3}\left(t, E_{2}\right)\right\| \leq \lambda_{3}\left\|E(t)-E\left(t_{2}\right)\right\|, \\
& \left\|p_{4}(t, I)-p_{4}\left(t, I_{1}\right)\right\| \leq \lambda_{4}\left\|I(t)-I\left(t_{1}\right)\right\|, \\
& \left\|p_{5}(t, T)-p_{5}\left(t, T_{1}\right)\right\| \leq \lambda_{5}\left\|T(t)-T\left(t_{1}\right)\right\|, \\
& \left\|p_{6}(t, R)-p_{6}\left(t, R_{1}\right)\right\| \leq \lambda_{6}\left\|R(t)-R\left(t_{1}\right)\right\| .
\end{aligned}
$$

Rewrite model (15) in recursive form as follows,

$$
\begin{aligned}
S_{n}(t)= & \frac{(1-\alpha)}{A B(\alpha)} p_{1}\left(t, S_{n-1}\right)+\frac{\alpha}{A B(\alpha) \Gamma(\alpha)} \int_{0}^{t} p_{1}\left(\xi, S_{n-1}\right)(t-\xi)^{\alpha-1} d \xi \\
E_{1 n}(t)= & \frac{(1-\alpha)}{A B(\alpha)} p_{2}\left(t, E_{1 n-1}\right)+\frac{\alpha}{A B(\alpha) \Gamma(\alpha)} \int_{0}^{t} p_{2}\left(\xi, E_{1 n-1}\right)(t-\xi)^{\alpha-1} d \xi \\
E_{2 n}(t)= & \frac{(1-\alpha)}{A B(\alpha)} p_{3}\left(t, E_{2 n-1}\right)+\frac{\alpha}{A B(\alpha) \Gamma(\alpha)} \int_{0}^{t} p_{3}\left(\xi, E_{2 n-1}\right)(t-\xi)^{\alpha-1} d \xi \\
I_{n}(t)= & \frac{(1-\alpha)}{A B(\alpha)} p_{4}\left(t, I_{n-1}\right)+\frac{\alpha}{A B(\alpha) \Gamma(\alpha)} \int_{0}^{t} p_{4}\left(\xi, I_{n-1}\right)(t-\xi)^{\alpha-1} d \xi \\
T_{n}(t)= & \frac{(1-\alpha)}{A B(\alpha)} p_{5}\left(t, T_{n-1}\right)+\frac{\alpha}{A B(\alpha) \Gamma(\alpha)} \int_{0}^{t} p_{5}\left(\xi, T_{n-1}\right)(t-\xi)^{\alpha-1} d \xi \\
R_{n}(t)= & \frac{(1-\alpha)}{A B(\alpha)} p_{6}\left(t, R_{n-1}\right)+\frac{\alpha}{A B(\alpha) \Gamma(\alpha)} \int_{0}^{t} p_{6}\left(\xi, R_{n-1}\right)(t-\xi)^{\alpha-1} d \xi
\end{aligned}
$$

Moreover, the initial conditions are,

$S_{0}(t)=S(0), E_{10}(t)=E(0), E_{20}(t)=E(0), I_{0} \cdot(t)=I(0), . T_{0}(t)=T(0),, . R_{0}(t)=R(0)$.

For the difference of successive terms, we get the following results:

$$
\begin{aligned}
& \varphi_{1 n}(t)=S_{n}(t)-S_{n-1}(t)=\frac{(1-\alpha)}{A B(\alpha)}\left\{p_{1}\left(t, S_{n-1}\right)-p_{1}\left(t, S_{n-2}\right)\right\} \\
& +\frac{\alpha}{A B(\alpha) \Gamma(\alpha)} \int_{0}^{t}(t-\xi)^{\alpha-1}\left\{p_{1}\left(\xi, S_{n-1}\right)-p_{1}\left(\xi, S_{n-2}\right)\right\} d \xi, \\
& \varphi_{2 n}(t)=E_{1 n}(t)-E_{1 n-1}(t)=\frac{(1-\alpha)}{A B(\alpha)}\left\{p_{2}\left(t, E_{1 n-1}\right)-p_{2}\left(t, E_{1 n-2}\right)\right\} \\
& +\frac{\alpha}{A B(\alpha) \Gamma(\alpha)} \int_{0}^{t}(t-\xi)^{\alpha-1}\left\{p_{2}\left(\xi, E_{1 n-1}\right)-p_{2}\left(\xi, E_{1 n-2}\right)\right\} d \xi,
\end{aligned}
$$




$$
\begin{gathered}
\varphi_{3 n}(t)=E_{2 n}(t)-E_{2 n-1}(t)=\frac{(1-\alpha)}{A B(\alpha)}\left\{p_{3}\left(t, E_{2 n-1}\right)-p_{3}\left(t, E_{2 n-2}\right)\right\} \\
+\frac{\alpha}{A B(\alpha) \Gamma(\alpha)} \int_{0}^{t}(t-\xi)^{\alpha-1}\left\{p_{3}\left(\xi, E_{2 n-1}\right)-p_{3}\left(\xi, E_{2 n-2}\right)\right\} d \xi \\
\varphi_{4 n}(t)=I_{n}(t)-I_{n-1}(t)=\frac{(1-\alpha)}{A B(\alpha)}\left\{p_{4}\left(t, I_{n-1}\right)-p_{4}\left(t, I_{n-2}\right)\right\} \\
+\frac{\alpha}{A B(\alpha) \Gamma(\alpha)} \int_{0}^{t}(t-\xi)^{\alpha-1}\left\{p_{4}\left(\xi, I_{n-1}\right)-p_{4}\left(\xi, I_{n-2}\right)\right\} d \xi \\
\varphi_{5 n}(t)=T_{n}(t)-T_{n-1}(t)=\frac{(1-\alpha)}{A B(\alpha)}\left\{p_{5}\left(t, T_{n-1}\right)-p_{5}\left(t, T_{n-2}\right)\right\} \\
+\frac{\alpha}{A B(\alpha) \Gamma(\alpha)} \int_{0}^{t}(t-\xi)^{\alpha-1}\left\{p_{5}\left(\xi, T_{n-1}\right)-p_{5}\left(\xi, T_{n-2}\right)\right\} d \xi \\
\varphi_{6 n}(t)=R_{n}(t)-R_{n-1}(t)=\frac{(1-\alpha)}{A B(\alpha)}\left\{p_{6}\left(t, R_{n-1}\right)-p_{6}\left(t, R_{n-2}\right)\right\} \\
+\alpha(\alpha)
\end{gathered}
$$

Consider,

$$
\begin{aligned}
S_{n}(t) & =\sum_{i=1}^{n} \varphi_{1 i}(t), \\
E_{1 n}(t) & =\sum_{i=1}^{n} \varphi_{2 i}(t), \\
E_{2 n}(t) & =\sum_{i=1}^{n} \varphi_{3 i}(t), \\
I_{n}(t) & =\sum_{i=1}^{n} \varphi_{4 i}(t), \\
T_{n}(t) & =\sum_{i=1}^{n} \varphi_{5 i}(t), \\
R_{n}(t) & =\sum_{i=1}^{n} \varphi_{6 i}(t) .
\end{aligned}
$$

Using the norm on (16), the triangle inequality, and the Lipchitz condition given in (13), we get.

$$
\left\|\phi_{1 n}(t)\right\| \leq \frac{(1-\alpha)}{A B(\alpha)} \lambda_{1}\left\|\phi_{1(n-1)}(t)\right\|+\frac{\alpha}{A B(\alpha)+\Gamma(\alpha)} \lambda_{1} \int_{0}^{t}\left\|\phi_{1(n-1)}(y)\right\| d y .
$$


After applying the same method to the remaining equations, we get

$$
\begin{aligned}
\left\|\phi_{2 n}(t)\right\| & \leq \frac{(1-\alpha)}{A B(\alpha)} \lambda_{2}\left\|\phi_{2(n-1)}(t)\right\|+\frac{\alpha}{A B(\alpha)+\Gamma(\alpha)} \lambda_{2} \int_{0}^{t}\left\|\phi_{2(n-1)}(y)\right\| d y \\
\left\|\phi_{3 n}(t)\right\| & \leq \frac{(1-\alpha)}{A B(\alpha)} \lambda_{3}\left\|\phi_{3(n-1)}(t)\right\|+\frac{\alpha}{A B(\alpha)+\Gamma(\alpha)} \lambda_{3} \int_{0}^{t}\left\|\phi_{3(n-1)}(y)\right\| d y \\
\left\|\phi_{4 n}(t)\right\| & \leq \frac{(1-\alpha)}{A B(\alpha)} \lambda_{4}\left\|\phi_{4(n-1)}(t)\right\|+\frac{\alpha}{A B(\alpha)+\Gamma(\alpha)} \lambda_{4} \int_{0}^{t}\left\|\phi_{4(n-1)}(y)\right\| d y \\
\left\|\phi_{5 n}(t)\right\| & \leq \frac{(1-\alpha)}{A B(\alpha)} \lambda_{5}\left\|\phi_{5(n-1)}(t)\right\|+\frac{\alpha}{A B(\alpha)+\Gamma(\alpha)} \lambda_{5} \int_{0}^{t}\left\|\phi_{5(n-1)}(y)\right\| d y \\
\left\|\phi_{6 n}(t)\right\| & \leq \frac{(1-\alpha)}{A B(\alpha)} \lambda_{6}\left\|\phi_{6(n-1)}(t)\right\|+\frac{\alpha}{A B(\alpha)+\Gamma(\alpha)} \lambda_{6} \int_{0}^{t}\left\|\phi_{6(n-1)}(y)\right\| d y
\end{aligned}
$$

In light of the above result, the following theorem is established.

Theorem 5. The solution of the TB fractional model exists and unique under the conditions that there exist some $t_{0}$, such that,

$$
\frac{(1-\alpha)}{A B(\alpha)} \lambda_{1}+\frac{t_{0} \alpha}{A B(\alpha) \Gamma(\alpha)} \lambda_{2}<1, \text { for } i=1,2, . ., 5
$$

Proof. As we know that $S(t), E_{1}(t), E_{2}(t), I(t), T(t)$ and $R(t)$ satisfy Lipchitz condition and are bounded functions. As a result of using equations (18) and (19), the following relationship is obtained.

$$
\begin{aligned}
& \left\|\phi_{1 n}(t)\right\| \leq\left\|S_{n}(t)\right\|\left[\frac{(1-\alpha)}{A B(\alpha)} \lambda_{1}+\frac{t_{0} \alpha}{A B(\alpha) \Gamma(\alpha)} \lambda_{1}\right]^{n}, \\
& \left\|\phi_{2 n}(t)\right\| \leq\left\|E_{1 n}(t)\right\|\left[\frac{(1-\alpha)}{A B(\alpha)} \lambda_{2}+\frac{t_{0} \alpha}{A B(\alpha) \Gamma(\alpha)} \lambda_{2}\right]^{n}, \\
& \left\|\phi_{3 n}(t)\right\| \leq\left\|E_{2 n}(t)\right\|\left[\frac{(1-\alpha)}{A B(\alpha)} \lambda_{3}+\frac{t_{0} \alpha}{A B(\alpha) \Gamma(\alpha)} \lambda_{3}\right]^{n}, \\
& \left\|\phi_{4 n}(t)\right\| \leq\left\|I_{n}(t)\right\|\left[\frac{(1-\alpha)}{A B(\alpha)} \lambda_{4}+\frac{t_{0} \alpha}{A B(\alpha) \Gamma(\alpha)} \lambda_{4}\right]^{n}, \\
& \left\|\phi_{5 n}(t)\right\| \leq\left\|T_{n}(t)\right\|\left[\frac{(1-\alpha)}{A B(\alpha)} \lambda_{5}+\frac{t_{0} \alpha}{A B(\alpha) \Gamma(\alpha)} \lambda_{5}\right]^{n}, \\
& \left\|\phi_{6 n}(t)\right\| \leq\left\|R_{n}(t)\right\|\left[\frac{(1-\alpha)}{A B(\alpha)} \lambda_{6}+\frac{t_{0} \alpha}{A B(\alpha) \Gamma(\alpha)} \lambda_{6}\right]^{n} .
\end{aligned}
$$

The above-mentioned solutions' existence and continuity are proved. We define the following functions to prove that the aforementioned functions form a solution for the TB model (9), 


$$
\begin{gathered}
S(t)-S(0 .)=S_{n}(t)-\beta_{1 n}(t), \\
E_{1}(t)-E_{2}(0)=E_{1 n}(t)-\beta_{2 n}(t), \\
E_{2}(t)-E_{2}(0)=E_{2 n}(t)-\beta_{3 n}(t), \\
I(t)-I(0)=I_{n}(t)-\beta_{4 n}(t), \\
T(t)-T(0)=T_{n}(t)-\beta_{5 n}(t), \\
R(t)-R(0)=R_{n}(t)-\beta_{6 n}(t) .
\end{gathered}
$$

Further, we get,

$$
\begin{aligned}
\left\|\beta_{1 n}(t)\right\| & =\left\|\frac{(1-\alpha)}{A B(\alpha)}\left\{p_{1}(t, S)-p_{1}\left(t, S_{n-1}\right)\right\}+\frac{\alpha}{A B(\alpha) \Gamma(\alpha)} \times \int_{0}^{t}(t-\xi)^{\alpha-1}\left\{p_{1}(\xi, S)-p_{1}\left(\xi, S_{n-1}\right)\right\} d \xi\right\| \\
& \leq \frac{1-\alpha}{A B(\alpha)}\left\|p_{1}(t, S)-p_{1}\left(t, S_{n-1}\right)\right\|+\frac{\alpha}{A B(\alpha) \Gamma(\alpha)} \int_{0}^{t}\left\|\left\{p_{1}(\xi, S)-p_{1}\left(\xi, S_{n-1}\right)\right\}\right\| d \xi \\
& \leq \frac{(1-\alpha)}{A B(\alpha)} \lambda_{1}\left\|S-S_{n-1}\right\|+\frac{\alpha}{A B(\alpha) \Gamma(\alpha)} \lambda_{1}\left\|S-S_{n-1}\right\| t .
\end{aligned}
$$

When we repeated the procedure at $t_{0}$, we got the following results,

$$
\left\|\beta_{1 n}(t)\right\| \leq\left(\frac{1-\alpha}{A B(\alpha)}+\frac{\alpha t_{0}}{A B(\alpha) \Gamma(\alpha)}\right)^{n+1} \lambda_{1}^{n+1} M
$$

Taking limit of equation (23) as $n$ approaches to $\infty$, then clearly $\left\|\beta_{1 n}(t)\right\|$ tends to 0 . By the same analysis, we have $\left\|\beta_{2 n}(t)\right\|,\left\|\beta_{3 n}(t)\right\|,\left\|\beta_{4 n}(t)\right\|,\left\|\beta_{5 n}(t)\right\|$ and $\left\|\beta_{6 n}(t)\right\|$ tend to 0 whenever $n$ tends to $\infty$.

\section{Uniqueness of the solution}

Previously, we used fixed point theory to prove the existence of a solution for model (9). Following that, we provide the solution's uniqueness and give the following statement.

Theorem 6. The solution of the non-integer order derivative model given by (9) possesses a unique solution, whenever, the assumption holds,

$$
\left(1-\frac{1-\alpha}{A B(\alpha)} \lambda_{1}-\frac{\alpha}{A B(\alpha) \Gamma(\alpha)} \lambda_{1} t\right) \geq 0
$$

Proof. We show that the arbitrary order derivative model (9) is unique by considering that another set of solutions, such as $S_{1}(t), E_{1}(t), E_{2}(t), I_{1}(t), T_{1}(t)$ and $R_{1}(t)$ then,

$$
S(t)-S_{1}(t)=\frac{1-\sigma}{A B(\sigma)}\left(p_{1}(t, S)-p_{1}\left(t, S_{1}\right)\right)+\frac{\sigma}{A B(\sigma) \Gamma(\sigma)} \int_{0}^{t}\left(p_{1}(\xi, S)-p_{1}\left(\xi, S_{1}\right)\right) d \xi
$$


Applying norm on equation (20), it follows,

$$
\begin{aligned}
& \left\|S(t)-S_{1}(t)\right\| \\
& =\left\|\frac{1-\alpha}{A B(\alpha)}\left(p_{1}(t, S)-p_{1}\left(t, S_{1}\right)\right)+\frac{\alpha}{A B(\alpha) \Gamma(\alpha)} \int_{0}^{t}\left(p_{1}(\xi, S)-p_{1}\left(\xi, S_{1}\right)\right) d \xi\right\| \\
& \leq \frac{1-\alpha}{A B(\alpha)} \lambda_{1}\left\|S(t)-S_{1}(t)\right\|+\frac{\alpha}{A B(\alpha) \Gamma(\alpha)} \lambda_{1} t\left\|S(t)-S_{1}(t)\right\| .
\end{aligned}
$$

Which gives,

$$
\left\|S(t)-S_{1}(t)\right\|\left(1-\frac{1-\alpha}{A B(\alpha)} \lambda_{1}-\frac{\alpha}{A B(\alpha) \Gamma(\alpha)} \lambda_{1} t\right) \leq 0
$$

Clearly, $S(t)=S_{1}(t)$, if condition (24) holds. Similarly, $E(t)=E_{1}(t), E(t)=E_{2}(t), I(t)=I_{1}(t), T(t)=T_{1}(t)$ and $R(t)=R_{1}(t)$. Hence, the solution is unique.

\section{Numerical approximations}

The TB fractional model (9) numerical results are obtained here. To do this, we must first express the noninteger order derivative model given by (9) in fractional Volterra type before applying calculus results. The modified Adams Bash forth rule for the $\mathrm{AB}$ fractional integral operator is applied to derive the scheme for the TB model (9), The non-integer order derivative model (9) with AB derivative is obtained by applying the calculus result to the first equation of the TB model (9) as shown,

$$
S(t)-S(0)=\frac{1-\alpha}{A B(\alpha)} p_{1}(t, S)+\frac{\alpha}{A B(\alpha) \Gamma(\alpha)} \int_{0}^{t} p_{1}(\xi, S)(t-\xi)^{\alpha-1} d \xi
$$

$\operatorname{Over}\left[t_{p}, t_{p+1}\right]$, the function $p_{1}(\tau, S)$ can be approximated by the interpolation polynomial

$$
\begin{aligned}
M_{p}(\tau) & =\frac{\tau-t_{p-1}}{t_{p}-t_{p-1}} g\left(t_{p}, y\left(t_{p}\right)\right)-\frac{\tau-t_{p-1}}{t_{k}-t_{p-1}} g\left(t_{p-1}, y\left(t_{p-1}\right)\right) \\
& =\frac{g\left(t_{p}, y\left(t_{p}\right)\right)}{h}\left(\tau-t_{p-1}\right)-\frac{g\left(t_{p-1}, y\left(t_{p-1}\right)\right)}{h}\left(\tau-t_{p}\right) \\
& \cong \frac{g\left(t_{p}, y_{p}\right)}{h}\left(\tau-t_{p-1}\right)-\frac{g\left(t_{p-1}, y_{p-1}\right)}{h}\left(\tau-t_{p}\right) .
\end{aligned}
$$

Which gives, 


$$
\begin{aligned}
S_{n+1}= & S_{0}+\frac{1-\alpha}{A B(\alpha)} p_{1}\left(t_{n}, S\right)+\frac{\alpha}{A B(\alpha) \times \Gamma(\alpha)} \\
& \left(\sum_{p=o}^{n}\left[\frac{p_{1}\left(t_{k}, S\right)}{h} \int_{t_{p}}^{t_{p+1}}\left(\tau-t_{p-1}\right)\left(t_{n+1}-\tau\right)^{\alpha-1} d \tau-\frac{p_{1}\left(t_{p-1}, S\right)}{h} \int_{t_{p}}^{t_{p+1}}\left(\tau-t_{p}\right)\left(t_{n+1}-\tau\right)^{\alpha-1} d \tau\right]\right),
\end{aligned}
$$

Now,

$$
A_{\alpha, 1}=\int_{t_{p}}^{t_{p+1}}\left(\tau-t_{p-1}\right)\left(t_{n+1}-\tau\right)^{\alpha-1} d \tau
$$

and,

$$
A_{\alpha, 2}=\int_{t_{p}}^{t_{p+1}}\left(\tau-t_{p}\right)\left(t_{n+1}-\tau\right)^{\alpha-1} d \tau
$$

We get the following result when we calculate these integrals

$$
\begin{aligned}
& A_{\alpha, 1}=h^{\alpha+1} \frac{(n+1-p)^{\alpha}(n-p+2+\alpha)-(n-p)^{\alpha}(n-p+2+2 \alpha)}{\alpha(\alpha+1)}, \\
& A_{\alpha, 2}=h^{\alpha+1} \frac{(n+1-p)^{\alpha}-(n-p)^{\alpha}(n-p+1+\alpha)}{\alpha(\alpha+1)} .
\end{aligned}
$$

Finally,

$$
\begin{aligned}
& S_{n+1}=S_{0}+\frac{1-\alpha}{A B(\alpha)} p_{1}\left(t_{n}, S\right)+\frac{\alpha}{A B(\alpha) \times \Gamma(\alpha)} \\
& \sum_{p=0}^{n}\left[\begin{array}{l}
\frac{h^{\alpha} p_{1}\left(t_{p}, S\right)}{\Gamma(\alpha+2)}\left((n+1-p)^{\alpha}(n-p+2+\alpha)-(n-p)^{\alpha}(n-p+2+2 \alpha)\right) \\
-\frac{h^{\alpha} p_{1}\left(t_{p-1}, S\right)}{\Gamma(\alpha+2)}\left[(n+1-p)^{\alpha+1}-(n-p)^{\alpha}(n-p+1+\alpha)\right]
\end{array}\right] .
\end{aligned}
$$

From the remaining equations of the system (9), we obtained the recursive formulae follows as, 


$$
\begin{aligned}
& E_{1 n+1}=E_{0}+\frac{1-\alpha}{A B(\alpha)} p_{2}\left(t_{n}, E_{1}\right)+\frac{\alpha}{A B(\alpha) \Gamma(\alpha)} \\
& \sum_{p=0}^{n}\left(\begin{array}{l}
\left.\frac{h^{\alpha} p_{2}\left(t_{p}, E_{1}\right)}{\Gamma(\alpha+2)}\left((n+1-p)^{\alpha}(n-p+2+\alpha)-(n-p)^{\alpha}(n-p+2+2 \alpha)\right)\right) \\
-\frac{h^{\alpha} p_{2}\left(t_{p-1}, E_{1}\right)}{\Gamma(\alpha+2)}\left((n+1-p)^{\alpha+1}-(n-p)^{\alpha}(n-p+1+\alpha)\right)
\end{array}\right),
\end{aligned}
$$

$$
E_{2 n+1}=E_{0}+\frac{1-\alpha}{A B(\alpha)} p_{3}\left(t_{n}, E_{2}\right)+\frac{\alpha}{A B(\alpha) \Gamma(\alpha)}
$$$$
\sum_{p=0}^{n}\left(\begin{array}{l}
\left.\frac{h^{\alpha} p_{3}\left(t_{p}, E_{2}\right)}{\Gamma(\alpha+2)}\left((n+1-p)^{\alpha}(n-p+2+\alpha)-(n-p)^{\alpha}(n-p+2+2 \alpha)\right)\right) \\
-\frac{h^{\alpha} p_{3}\left(t_{p-1}, E_{2}\right)}{\Gamma(\alpha+2)}\left((n+1-p)^{\alpha+1}-(n-p)^{\alpha}(n-p+1+\alpha)\right)
\end{array}\right),
$$

$$
\begin{aligned}
& I_{n+1}=I_{0}+\frac{1-\alpha}{A B(\alpha)} p_{4}\left(t_{n}, I\right)+\frac{\alpha}{A B(\alpha) \Gamma(\alpha)} \\
& \sum_{p=0}^{n}\left(\begin{array}{l}
\frac{h^{\alpha} p_{4}\left(t_{p}, I\right)}{\Gamma(\alpha+2)}\left((n+1-p)^{\alpha}(n-p+2+\alpha)-(n-p)^{\alpha}(n-p+2+2 \alpha)\right) \\
-\frac{h^{\alpha} p_{4}\left(t_{p-1}, I\right)}{\Gamma(\alpha+2)}\left((n+1-p)^{\alpha+1}-(n-p)^{\alpha}(n-p+1+\alpha)\right)
\end{array}\right),
\end{aligned}
$$

$$
\begin{aligned}
& T_{n+1}=T_{0}+\frac{1-\alpha}{A B(\alpha)} p_{5}\left(t_{n}, T\right)+\frac{\alpha}{A B(\alpha) \Gamma(\alpha)} \\
& \sum_{p=0}^{n}\left(\begin{array}{l}
\left.\frac{h^{\alpha} p_{5}\left(t_{p}, T\right)}{\Gamma(\alpha+2)}\left((n+1-p)^{\alpha}(n-p+2+\alpha)-(n-p)^{\alpha}(n-p+2+2 \alpha)\right)\right) \\
-\frac{h^{\alpha} p_{5}\left(t_{p-1}, T\right)}{\Gamma(\alpha+2)}\left((n+1-p)^{\alpha+1}-(n-p)^{\alpha}(n-p+1+\alpha)\right)
\end{array}\right),
\end{aligned}
$$

$$
\begin{aligned}
& R_{n+1}=R_{0}+\frac{1-\alpha}{A B(\alpha)} p_{6}\left(t_{n}, R\right)+\frac{\alpha}{A B(\alpha) \Gamma(\alpha)} \\
& \sum_{p=0}^{n}\left(\begin{array}{l}
\frac{h^{\alpha} p_{6}\left(t_{p}, R\right)}{\Gamma(\alpha+2)}\left((n+1-p)^{\alpha}(n-p+2+\alpha)-(n-p)^{\alpha}(n-p+2+2 \alpha)\right) \\
-\frac{h^{\alpha} p_{6}\left(t_{p-1}, R\right)}{\Gamma(\alpha+2)}\left((n+1-p)^{\alpha+1}-(n-p)^{\alpha}(n-p+1+\alpha)\right)
\end{array}\right) .
\end{aligned}
$$




\section{Simulation results}

After the successful execution of modified Adams Bashforth numerical result on the TB model (9), we obtained the graphical results of the fractional order TB model (9), by considering and assigning values of fractional parameter $\alpha, \beta$ and $\gamma \in[0,1]$. The time interval in the graphical results is kept as 300 units. The parameters used in the graphical results are projected based on the available TB data from NTP Pakistan. The graphical behavior of the model (9) and the effect of $\alpha$ transmission rate on the susceptible, exposed, infected, treated and recovered classes for $\alpha=0.01,0.90$ is shown in Figure 1, likewise, the effect of $\beta$ transmission on the exposed, infected and recovered individuals for $\alpha=0.01,0.90$ is given in Figure 2 .

Table 1: Parameters setting for the TB model (9).

\begin{tabular}{|c|c|c|c|}
\hline Parameter & Description & Baseline value & Reference \\
\hline $\bar{\beta} \beta$ & Contact rate & 0.6001 & "Fitted \\
\hline$\alpha$ & Recovery rate & 0.0100 & Fitted \\
\hline $\bar{\gamma}$ & $\begin{array}{l}\text { Treatment rate of } \\
\text { infective individuals }\end{array}$ & 0.1500 & Fitted \\
\hline$\overline{\mu \mu}$ & Natural mortality rate & $1 / 67.7$ & $\begin{array}{l}\text { Pakistan Bureau of Statistics } \\
\text { [5] }\end{array}$ \\
\hline$\alpha_{1}$ & $\begin{array}{l}\text { Disease induced death } \\
\text { rate in } I\end{array}$ & 0.2738 & Fitted \\
\hline$\Lambda$ & Birth rate & $450,862.20088$ & Estimated \\
\hline$\alpha_{2}$ & $\begin{array}{l}\text { Disease induced death } \\
\text { rate during treatment }\end{array}$ & 0.1000 & Fitted \\
\hline $\bar{\delta}$ & $\begin{array}{l}\text { Leaving rate of } \\
\text { treated individual re- } \\
\text { enter to } I \text { or } E_{1}\end{array}$ & 1.0649 & Fitted \\
\hline $\bar{\eta}$ & Failure of treatment & 0.2959 & Fitted \\
\hline$\varepsilon_{1}$ & $\begin{array}{l}\text { Rate of moving from } \\
E_{1} \text { to } E_{2}\end{array}$ & 0.2351 & Fitted \\
\hline$\varepsilon_{2}$ & $\begin{array}{l}\text { Transfer rate from } E_{2} \\
\text { to } I\end{array}$ & 0.2001 & Fitted \\
\hline$q$ & $\begin{array}{l}\text { Fraction of susceptible } \\
\text { individuals being } \\
\text { infected }\end{array}$ & 0.5259 & Fitted \\
\hline
\end{tabular}




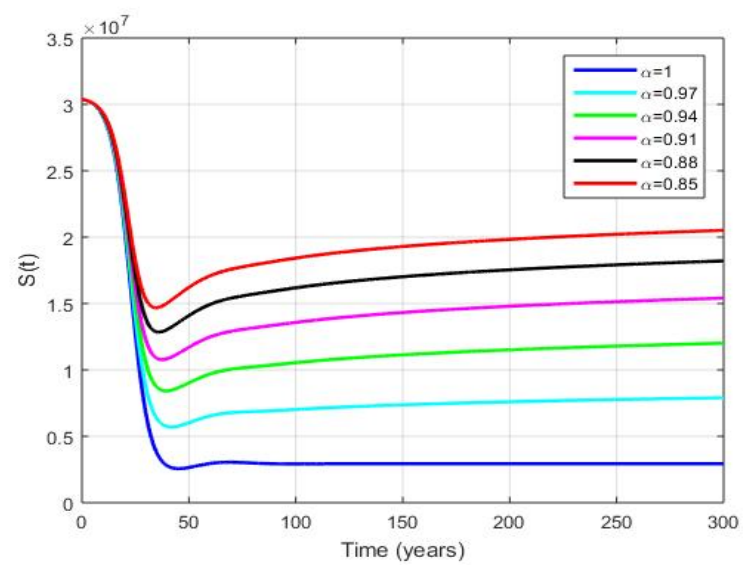

(a)

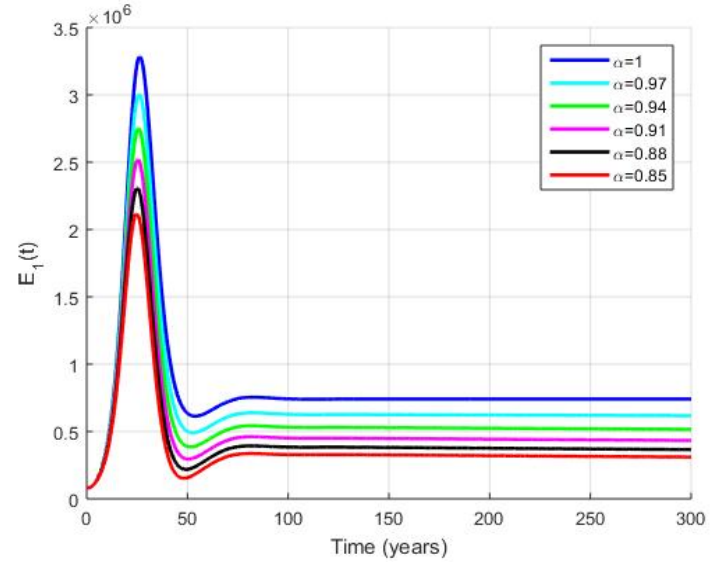

(b)

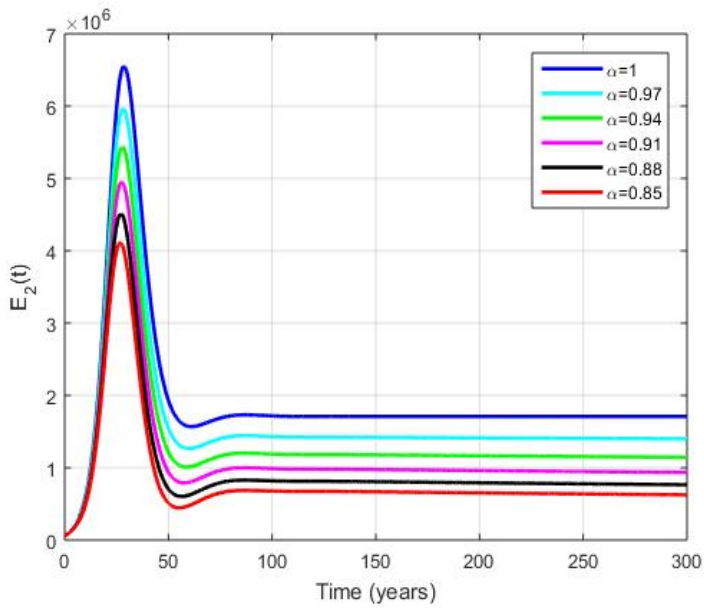

(c)

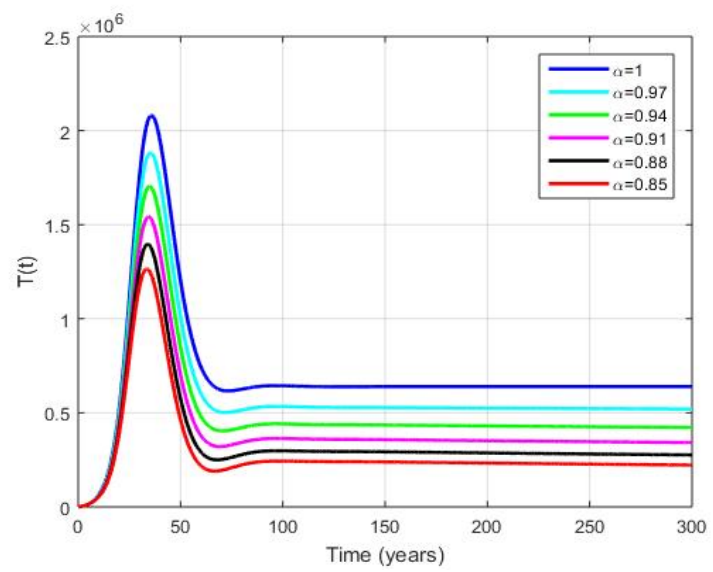

(e)

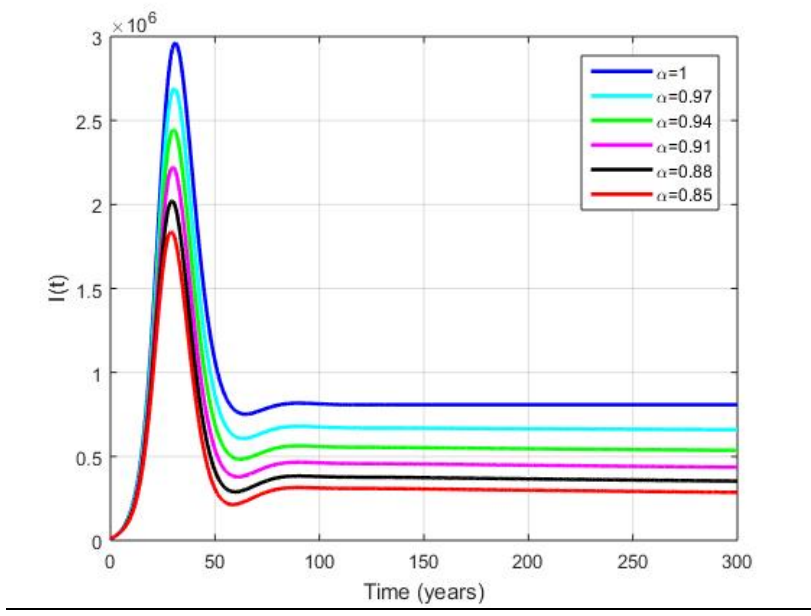

(d)

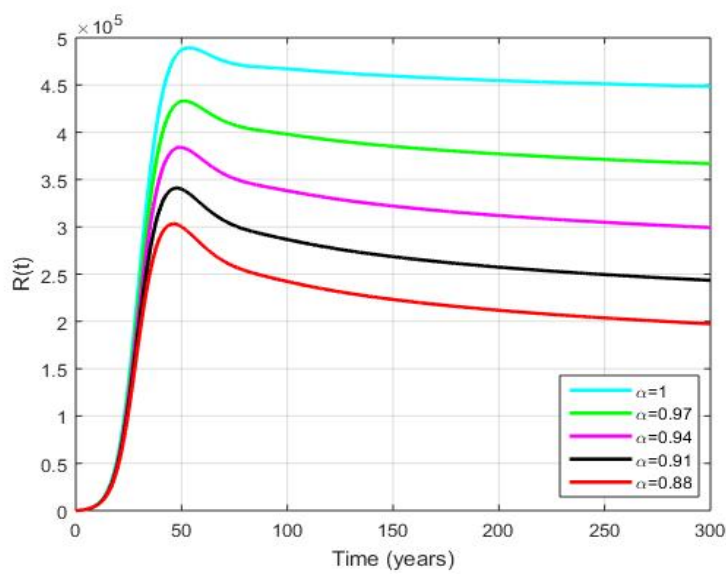

(f)

Fig. 1 Simulation of TB model (9) with AB derivative. 


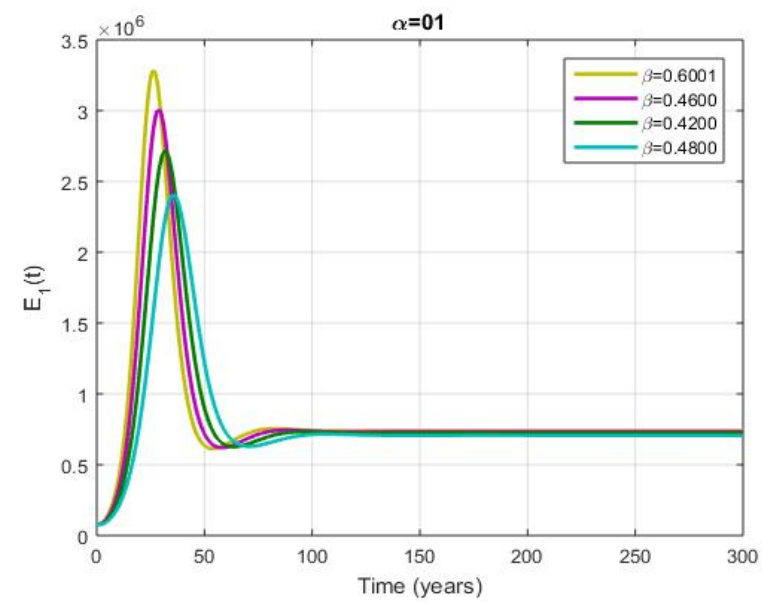

(a)

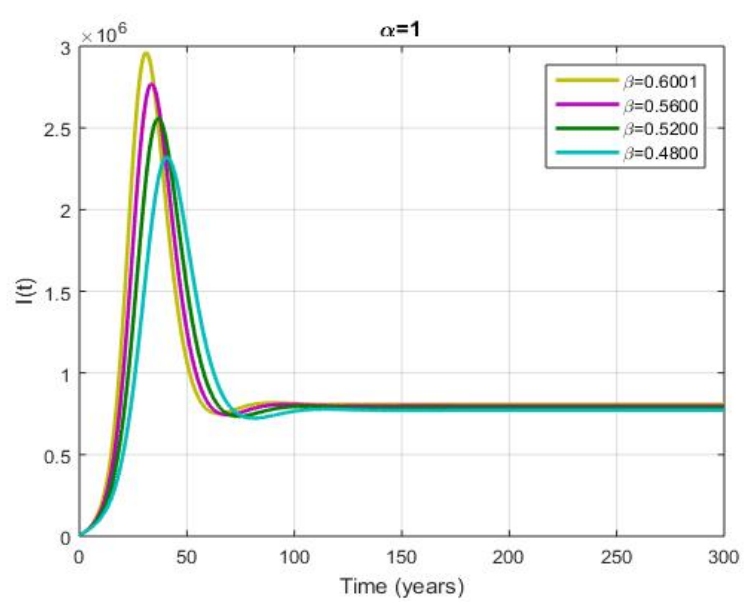

(c)

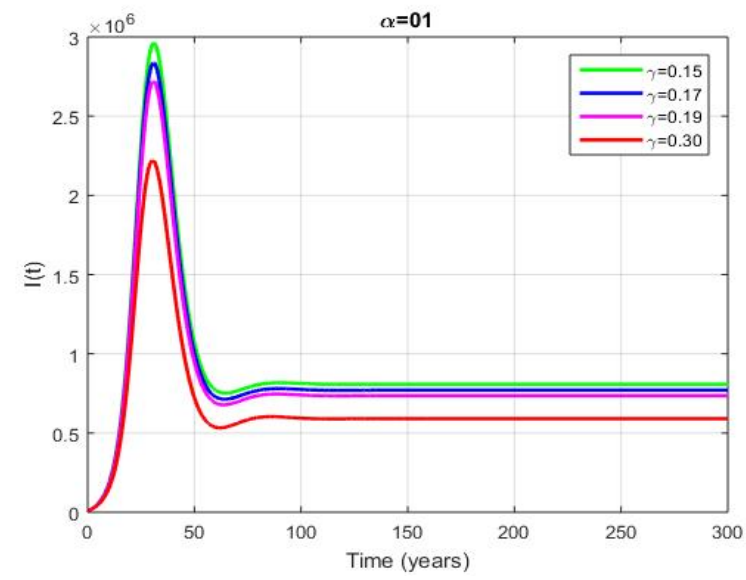

(e)

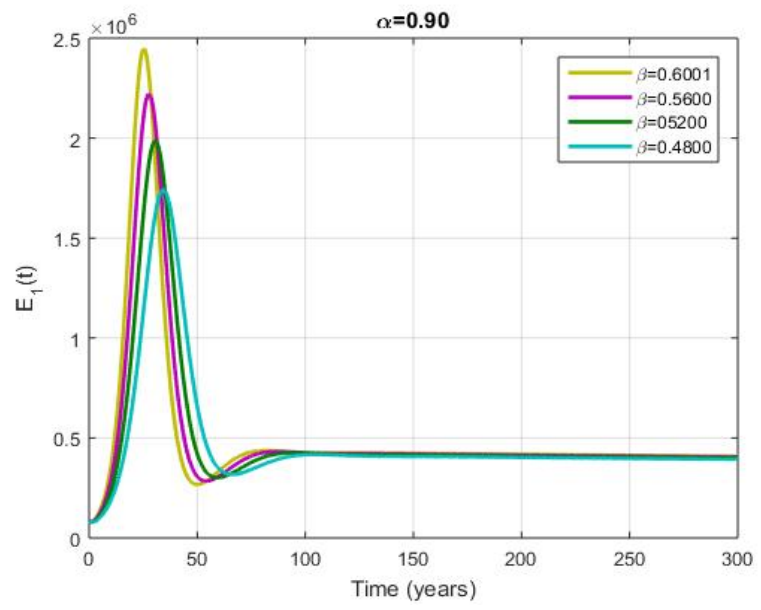

(b)

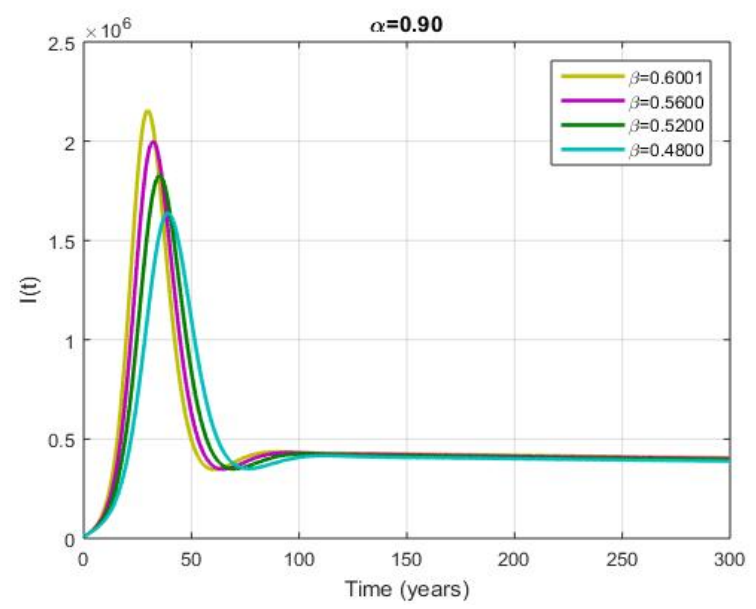

(d)

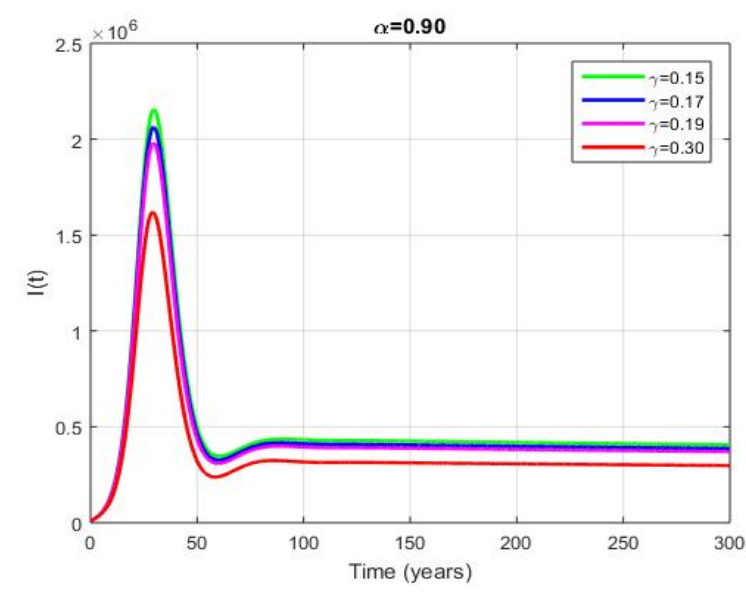

(f)

Fig. 2 Simulation of TB model (9) with AB derivative. 


\section{Conclusion}

We successfully analyzed a TB model with an AB derivative. Data from NTP Khyber Pakhtunkhwa, Pakistan, was used to parameterize model parameters utilized in numerical simulations from 2002 to 2017. We obtained numerical results by assuming the fractional parameter with different values and provided a comprehensive discussion. The essential results of the fractional order model have been analyzed and briefly described. An iterative method was used to find the solution to the TB model. Fixed theory was used to prove the model solution, uniqueness, and existence. It can be perceived from the graphical results that newly introduced derivative for the TB model provides flexible results that might be more useful as compared to integer order derivative. If $R_{0}<1$, then $Z_{0}$ is GAS. When $R_{0}>1$, it also possesses a unique $D_{1}$ and is Global asymptotic stable. Finally, numerical simulations of the model are shown to verify the theoretical results, to show the effects of model parameters and highlight the effect of TB infection.

\section{Competing Interests}

The authors declare no competing interests.

\section{References}

[1] World Health Organization Media Centre, 2018, https://www.who.int/en/news-room/factheets/detail/tuberculosis.

[2] World Health Organization. Global tuberculosis report, 2016. https://www.who.int/tb/country/data/profiles/en/

[3] Pakistan Bureau of Statistics. Pakistan's 6th census: population of major cities census, http://www.pbscensus.gov.pk/

[4] Y. Yang, J. Li, Z. Ma and L. Liu, Global stability of two models with incomplete treatment for tuberculosis , Chaos, Solitons \& Fractals, 43, 79 - 85 (2010).

[5] J. Liu and T. Zhang, Global stability for a tuberculosis model, Math. Comp. Modelling, 54, 836 - 845 (2011)

[6] L. Liu, X. Q. Zhao and Y. Zhou, A tuberculosis model with seasonality, Chao.Soliton.Fract.72, 931-952 (2010).

[7] S. R. Wallis, Mathematical models of tuberculosis reactivation and relapse, Front. in Micro-biol. 7, 1-7 (2016).

[8] Zhang, X. H., Ali, A., Khan, M. A., Alshahrani, M. Y., Muhammad, T., \& Islam, S. (2021).

Mathematical Analysis of the TB Model with Treatment via Caputo-Type Fractional

Derivative. Discrete Dynamics in Nature and Society, 2021.

[9] Li, X. P., Gul, N., Khan, M. A., Bilal, R., Ali, A., Alshahrani, M. Y. \& Islam, S. (2021). A new Hepatitis B model in light of asymptomatic carriers and vaccination study through Atangana-Baleanu derivative. Results in Physics, 29, 104603.

[10] S. G. Samko, A. A. Kilbas, I. O. Marichev and others, Fractional Integrals and Derivatives, Gordon and Breach Science Publishers, Yverdon, 1993. 
[11] M. Caputo and M. Fabrizio, A new definition of fractional derivative without singular kernel,Progr.Fract. Differ. Appl, 2, 1-13 (2015).

[12] National TB Control Program Pakistan (NTP). http://www.ntp.gov.pk/webdatabase.php.

[13] Ali, A., Islam, S., Khan, M. R., Rasheed, S., Allehiany, F. M., Baili, J., ... \& Ahmad, H.

Dynamics of a fractional order Zika virus model with mutant. Alexandria Engineering Journal, 2021.

[14] Li, Y. X., Alshehri, M. G., Algehyne, E. A., Ali, A., Khan, M. A., Muhammad, T., \& Islam, S.

Fractional study of Huanglongbing model with singular and non-singular kernel. Chaos, Solitons \& Fractals, 148, 111037 (2021).

[15] Oud, M. A. A., Ali, A., Alrabaiah, H., Ullah, S., Khan, M. A., \& Islam, S. A fractional order mathematical model for COVID-19 dynamics with quarantine, isolation, and environmental viral load. Advances in Difference Equations. 2021(1), 1-19 (2021).

[16] Chu, Y. M., Ali, A., Khan, M. A., Islam, S., \& Ullah, S. (2021). Dynamics of fractional order COVID-19 model with a case study of Saudi Arabia. Results in Physics, 21, 103787.

[17] Ali, A., Alshammari, F. S., Islam, S., Khan, M. A., \& Ullah, S. Modeling and analysis of the dynamics of novel coronavirus (COVID-19) with Caputo fractional derivative. Results in Physics, 20, 103669 (2021).

[18] P. V. D. Driessche and J. Watmough, Reproduction number and sub-threshold endemic equi-libria for compartmental models of disease transmission, Math. Bios., 180, 29-48 (2002).

[19] Van den Driessche, P, Watmough, J. Reproduction numbers and sub-threshold endemic equilibria for compartmental models of disease transmission. Math Biosci. 180, 29-48 (2002).

[20] LaSalle, JP .The stability of dynamical systems, Vol. 25. Philadelphia, PA: SIAM, 1976.

[21] M. Toufik and A. Atangana, New numerical approximation of fractional with non-local and nonsingular kernel: Application to chaotic models, Chaos Solitons Fract. 132.

[22] Churchyard G, Kim P, Shah NS, Rustomjee R, Gandhi N, Mathema B, et al. What we know about tuberculosis transmission: an overview. J Infect Dis. 216(suppl_6), S629-35 (2017).

[23] A. Atangana and D. Baleanu, New fractional derivatives with nonlocal and non-singular kernel: theory and application to heat transfer model, Therm. Sci. 20, 763-769 (2016).

[24] Pauline Van den Driessche, James Watmough, Math. Biosci. 180, 29 (2002).

[25] Denis Matignon, Stability results for fractional differential equations with applications tocontrol processing, in Computational Engineering in Systems Applications (IMACS,

IEEE-SMC Lille, France). 2, 963-968 (1996). 